\title{
De la critique des patients
}

\section{Eberhard Wolff}

PD Dr rer. soc., rédacteur culture, histoire, société

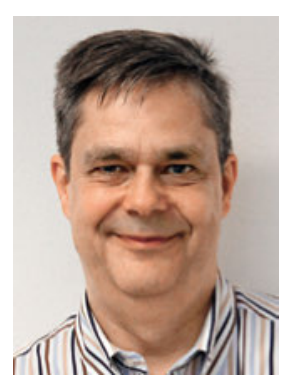

Références

1 Oertle D. Die Hausärzte sterben aus: na und? (Les médecins de famille sont en voie de disparition. Et alors?) Bulletin des méde cins suisses 2016;97(5152):1804-5.

2 Gesundheitskatechismus für das Landvolk und den gemeinen Mann. Berlin, Stettin: Nicolai; 1781 . Je remercie le Prof. Jutta Dornheim, de Brême, de l'avoir porté à mon attention.

3 Liek E. Les Méfaits des assurances sociales en Allemagne et les moyens d'y remédier. Paris: Editions Payot; 1929. V.a. Broghammer H. Der Danziger Arzt Erwin Liek (1878-1935). Herbolzheim Centaurus; 2000 et Jehs M. Erwin Liek. Welt anschauung und standespolitische Einstellung im Spiegel seiner Schriften. Francfort-sur-le-Main: Mabuse; 1994.

4 Sonderegger JL. Vorposten der Gesundheitspflege. $5^{e}$ édition, Berlin: Julius Springer; 1901, 569.

eberhard.wolff[at]saez.ch
Je me souviens d'une caricature qui m'avait amusé: dans un restaurant, deux serveurs chevronnés contemplent les tables coquettement mises et encore inoccupées. L'un dit à l'autre: «Et dans deux minutes, ils vont arriver et recommencer à tout salir.» Les cyniques affirment en effet que c'est sans clients ou sans passagers que les restaurants ou les compagnies aériennes fonctionnent le mieux.

Trois jours avant la fête de la paix dans le monde, un médecin de famille a lancé dans cette revue une "polémique» contre «Le Nouveau Patient» (LNP) [1] - faisant la joie de la plupart de ses collègues. La réprimande se présentait plus ou moins ainsi: LNP veut tout, tout de suite, sans fournir le moindre effort. Il est hypocondriaque et têtu, croit tout savoir, mais est mal informé. Il se montre ingrat et infidèle à l'égard du médecin; il ne s'occupe pas des coûts pour les caisses-maladie et manque donc de solidarité. LNP a, entre autres, été dévoyé par les cabinets sans rendez-vous.

Il faut toutefois noter que ces réprimandes des médecins contre «les nouveaux patients» (des deux sexes) sont un phénomène récurrent. Si on l'écrivait, l'«Histoire de la critique des patients» donnerait un pavé aussi volumineux que divertissant.

Adam Andreas Senfft (1740-1795), professeur de physiologie à Würzburg, qualifiait en 1781 la population rurale de «stupide», «aveugle», «naïve», «niaise», «ignorante» et «ingrate», dans un ouvrage qu'il lui destinait. Selon lui, ces patients étaient hypocondriaques. Ils n'avaient «aucune patience» et souhaitaient un «soulagement rapide», tout en attendant trop longtemps avant de consulter un médecin [2]. Les termes choisis nous semblent extrêmes, mais ne constituaient pas une exception à l'époque.

Les médecins d'alors espéraient d'ailleurs que de telles diatribes sous couvert d'éducation sanitaire auraient pour effet secondaire de rendre leur position sur le marché de la santé plus respectable.

Dans les années 1920, le vocabulaire de ces invectives extrêmes s'est surtout enrichi grâce au chirurgien ethno-nationaliste allemand Erwin Liek (1878-1935). Pour lui, les patients (assurés) étaient des "parasites». Liek leur diagnostiquait une dégénérescence, un ramollissement mental et une "déchéance morale» [3].

Spécialiste très applaudi à l'époque des attaques tous azimuts en politique de la santé, Liek en rejetait la faute sur le système de caisses-maladie. La critique des patients accompagnait donc l'offensive ciblée contre l'Etat social.

Chaque époque a ainsi ses propres diatribes contre les patients, qui se différencient par la patte de leur auteur et leur dureté. Mais un fil conducteur traverse leur histoire: le reproche que les patients ne pensent et n'agissent pas comme les médecins le leur demandent. Les raisons supposées de leur obstination évoluent cependant avec l'air du temps. Les applis du Web ont aujourd'hui remplacé les vieilles bonnes femmes d'antan; désormais, l'ennemi n'est plus le charlatan, mais le cabinet sans rendez-vous.

Senfft aussi s'indignait de la «désobéissance» des patients: un journalier souffrant d'occlusion intestinale et soigné gratuitement, par exemple, était «stupide et têtu», parce qu'au lieu des cataplasmes ramollissants prescrits par le médecin, il avait placé des sacs d'avoine sèche et chaude sur son ventre, sur les conseils d'une "vieille bonne femme». Commentaire de Senfft: "Je perdis l'envie de continuer à le soigner [...], car vraiment j'ai le défaut de ne plus pouvoir aimer les gens qui n'obéissent pas. [...] Il mourut, comme il se devait.» Du point de vue de l'éthique médicale, le moins que l'on puisse dire est que l'argumentation de la critique des patients peut très vite déraper.

Nous pouvons néanmoins nous représenter ce type de diatribes comme une tradition médicale et un art qui, sous différents atours, a mûri jusqu'à devenir un genre littéraire à part entière. Un ars longa, pour ainsi dire, ce qui nous amène à Hippocrate. Peut-être cet art devraitil par conséquent être repris dans un futur serment des médecins ou intégré à la formation continue.

La profession de médecin n'est pas seule dans ce cas. D'autres métiers ont leur propre variante: Peter Handke a par exemple élevé il y a près d'un demi-siècle «l'outrage au public» au rang d'art avec sa pièce de théâtre éponyme.

L'ancêtre de tous les présidents de la FMH, Jakob Laurenz Sonderegger (1825-1896), avait lui aussi bien du mal avec les patients, qui ne cessaient de le «déserter». Sonderegger renonça toutefois à les invectiver, déclarant: «Il n'y a rien de plus simple que les relations avec les patients: mets-toi à leur place et tu les comprendras.» Visiblement, il était conscient d'avoir, lui aussi, déjà sali quelques tables du restaurant médical. 